Artículos originales

ISSN 2519-0652

CASUS.2017;2(2):89-99.

\title{
Factores asociados a la calidad de vida profesional en enfermeros de un hospital público
}

\section{Zully Cazana-Vásquez ${ }^{1}$}

RESUMEN: Objetivo: determinar los factores asociados a la calidad de vida profesional en enfermeros de un hospital. Metodología: El estudio fue explicativo, transversal. La muestra estuvo conformada por 92 enfermeros. La variable dependiente fue la calidad de vida profesional. Se emplearon las prueba de Chi-cuadrado y de T student para el análisis bivariado. Para medir la fuerza de asociación se utilizó la regresión logística de poisson calculando razón de prevalencias. Resultados: el $51 \%$ tuvo una calidad alta. Los enfermeros que laboran en el servicio cirugía A tienen menos posibilidad de tener calidad alta (PR: 0.26). Por otra parte los que laboran en un turno matutino tienen más posibilidades de tener una carga laboral alta (PR: 30.38). Conclusiones: Laborar en el servicio cirugía A está asociado con la CVP global baja. Asimismo, se encontró asociaciones significativas entre los factores laborales y las dimensiones de la CVP global. Estos resultados contribuyen en el desarrollo de estrategias para mejorar los factores relacionados a la calidad de vida profesional del enfermero.

\footnotetext{
PALABRAS CLAVE: Calidad de Vida; Enfermeros; Estrategias nacionales.

Citar como: Cazana Z. Factores asociados a la calidad de vida profesional en enfermeros de un hospital público. CASUS. 2017;2(2):89-99.
} 


\section{INTRODUCCIÓN}

La calidad de vida profesional (CVP) definida como el sentimiento de bienestar que se deriva del equilibrio que el individuo percibe entre las demandas o cargas de la profesión y los recursos psicológicos, organizacionales y relacionales de que dispone para afrontar estas demandas (1), es un tema que viene siendo tratado en diversos ámbitos de estudios laborales especialmente para instituciones sanitarias puesto que se han encontrado altos niveles de estrés en el personal de salud $(1,2)$. Por ello, es primordial asegurar el mantenimiento del personal de salud y por ende garantizar la calidad de la atención (2).

Según la Organización Mundial de la Salud (OMS) hay 57 países que presentan un déficit crítico de personal sanitario (médicos, enfermeras y parteras), aproximadamente de 4,3 millones que no llegan al $80 \%$ de cobertura necesaria para la ejecución de intervenciones sustanciales (3). Esto conlleva a que el personal de salud esté atendiendo casi el doble o triple de pacientes dando origen a una carga laboral excesiva (3). Esta carga se asocia con una CVP baja, mientras que el buen apoyo directivo contribuye a una calidad de vida profesional alta (4). Cabe resaltar la CVP alta (88.4\%) en enfermeras que laboran en hospitales de Norteamérica (5) mientras que en Sudamérica fue $55 \%$ (6).

Existe una fuerte exigencia de que los profesionales de enfermería se capaciten para mejorar la calidad de vida de sus pacientes, sin embargo su propia calidad de vida laboral y necesidades son mayormente ignoradas. Es necesario reconocer que la calidad de vida profesional es esencial para optimizar el nivel de satisfacción tanto personal como profesional y por ende brindar un cuidado de calidad al paciente (7). En la actualidad estudios internacionales demuestran un desequilibrio entre el incremento profesional y la dotación de enfermería (8). Asimismo, se concluyó que los enfermeros con menor experiencia laboral poseen mayor cansancio emocional, despersonalización y estrés (9). En el Perú la migración del capital humano ha ido incrementando durante los últimos años (10). Otro estudio mostró que el agotamiento emocional prevalece más en los médicos que en los enfermeros debido a que estos últimos tienen una mayor motivación intrínseca (11). Pocos estudios demuestran que la CVP alta está directamente relacionada con un buen clima organizacional. El cual incluye el apoyo tanto de su equipo de trabajo como el de sus superiores. Un mal clima organizacional conllevaría a consecuencias negativas como sobrecargas y desmotivación laboral (12). Los enfermeros con más años de vida tienen menos quejas respecto a sus condiciones laborales, ya que la mayoría de ellos tienen un contrato seguro e incluso se sienten más motivados y autónomos por la experiencia laboral que han adquirido durante los años (13). Los profesionales de enfermería que laboran en turnos matutinos podrían tener problemas psicológicos como estrés y ansiedad somática debido a que estos horarios son más laboriosos por la cantidad de actividades (14). Los enfermeros con más antigüedad laboral en el hospital y con mayor tiempo de servicio perciben menos la carga laboral debido a su experiencia (15).

Teniendo en cuenta lo anterior, se muestra que la profesión de enfermería está expuesta a niveles de sobrecarga, en consecuencia esto afectaría su CVP. Sin embargo, hay pocos estudios que muestran qué factores están asociados a un nivel alto de CVP para disminuir en un futuro las exposiciones negativas y la insatisfacción del enfermero. Es importante no solo considerar a enfermeros que trabajan en servicios críticos sino también aquellos que trabajan en servicios de hospitalización donde se encuentran la mayor cantidad de pacientes con enfermedades terminales y contagiosas. A partir de lo anterior el estudio propuso determinar qué factores (sociodemográficos y laborales) están asociados a la calidad de vida laboral de enfermeros de un hospital público de Lima Norte.

\section{MATERIALES Y MÉTODOS}

El tipo de estudio fue descriptivo correlacional y explicativo de corte transversal. La muestra estuvo conformada por 92 licenciados de enfermería de un hospital público de Lima Norte. El estudio realizó un censo. Se incluyeron a profesionales de enfermería de los servicios de hospitalización. Se excluyeron a licenciados de enfermería con menos de 6 meses de trabajo en el Hospital, que tuvieran función administrativa o que laboraran en servicios críticos y quirúrgicos. 
La variable dependiente Calidad de vida profesional (CVP) fue medida por el cuestionario CVP-35 de 34 ítems validado a nivel internacional (14) y nacional (16). Cada ítem es valorado en una escala del 1 al 10, dividida en tres dimensiones: apoyo directivo (conformado por 12 ítems), categorizada en deficiente (12-24), regular (25-60), bueno (61-96) y excelente (97-120); cargas de trabajo (conformado por 11 ítems), categorizada en bajo (11-22), regular (23-55), alta (56-88) y excesiva (89-110) y motivación intrínseca (conformada por los diez ítems), categorizada en deficiente (10-20), regular (21-50), bueno (51-80) y excelente (81-100). Además, el cuestionario consta de un ítem que valora la calidad de vida profesional percibida categorizada en deficiente (1-2), regular (3-5), buena (6-8) y excelente (9-10). Asimismo, para calcular la CVP global se hizo la sumatoria de los ítems. La misma fue categorizada en deficiente (34-68), regular (69-170), buena (171-272) y excelente (273-340). Cabe resaltar que en el estudio se tomó en cuenta la categorización regular y buena tanto para CVP global como para las dimensiones debido a que los encuestados solo puntuaron los ítems de esas categorías.

Los factores se clasificaron en sociodemográficos y laborales. Respecto a los factores sociodemográficos fueron: sexo, variable categórica (masculino, femenino); edad, variable numérica; estado civil, categórica nominal (soltero, casado, viudo, divorciado, conviviente); número de hijos, variable numérica; lugar de residencia, variable categórica dicotómica (urbano, rural); tiempo que tarda en trasladarse desde su casa al hospital, variable numérica categorizada (menos o igual a $30 \mathrm{~min}, 31$ a 60 min, menos de 2 horas, más de 2 horas); horas de trabajo por día, variable numérica; años de experiencia laboral, variable numérica; años de experiencia en el hospital, variable numérica; años de experiencia en el servicio, variable numérica; condición laboral, variable categórica dicotómica (nombrado, contratado); sistema de turnos, variable categórica nominal (mixto, matutino, vespertino, nocturno); tiene otro trabajo, variable categórica dicotómica (sí, no); especialidad, variable categórica dicotómica (sí, no); diplomado, variable categórica dicotómica (sí, no); capacitación en el último año, variable categórica dicotómica (sí, no); es docente, variable categórica dicotómica (sí, no); servicio en que labora, variable categórica nominal (enfermedades infecciosas, gineco obstetricia, cirugía $\mathrm{A}$, cirugía $\mathrm{B}$, medicina $\mathrm{A}$, medicina $\mathrm{B}$; número de pacientes que atiende por turno, variable numérica; ingreso económico mensual, variable numérica.

Una vez recolectados los datos todos los análisis se realizaron empleando el programa estadístico Stata 13. En el análisis descriptivo de las variables categóricas se emplearon frecuencias y porcentajes. Así mismo, para las variables numéricas se utilizaron medidas de tendencia central y dispersión. Se emplearon las prueba de Chi-cuadrado y de T student para el análisis bivariado mediante tablas de contingencia. Para medir la fuerza de asociación se utilizó la regresión logística de poisson calculando razón de prevalencias (prevalence ratio, $\mathrm{PR}$ ) e intervalos de confianza al $95 \%$ con una significancia menor o igual a 0.05 .

La aprobación ética de este estudio fue realizada por un Comité de Ética. Asimismo, se garantizó la confidencialidad mediante el anonimato de los encuestados.

\section{RESULTADOS}

De los 92 enfermeros la edad media fue de 38.9 años y el 51\% tuvo un CVP alto. Asimismo, del total de los participantes, el $93.4 \%$ obtuvo una carga laboral regular, el $96.7 \%$ tuvo una motivación intrínseca buena, un $59.7 \%$ tuvo un apoyo directivo bueno y un $51.1 \%$ tuvo una regular calidad de vida profesional percibida. Respecto a los factores laborales, la mayoría de los enfermeros tuvo un tiempo de 31 minutos a una hora de traslado de su casa al hospital $(64.1 \%)$, son nombrados (55.4\%), tienen alguna especialidad $(72.8 \%)$, laboran en turnos mixtos (85.8\%), no cuentan con diplomado (86.9\%), trabajan en los servicios de Cirugía A y B, Medicina A y B. La media de años de experiencia en el hospital fue $9.6 \mathrm{y}$ en el servicio fue 6.4. Por último, la media de pacientes atendidos por turno fue $10 \mathrm{y}$ la media del ingreso económico fue 2889.1 soles (ver tabla 1). 
Tabla 1. Características descriptivas de los enfermeros

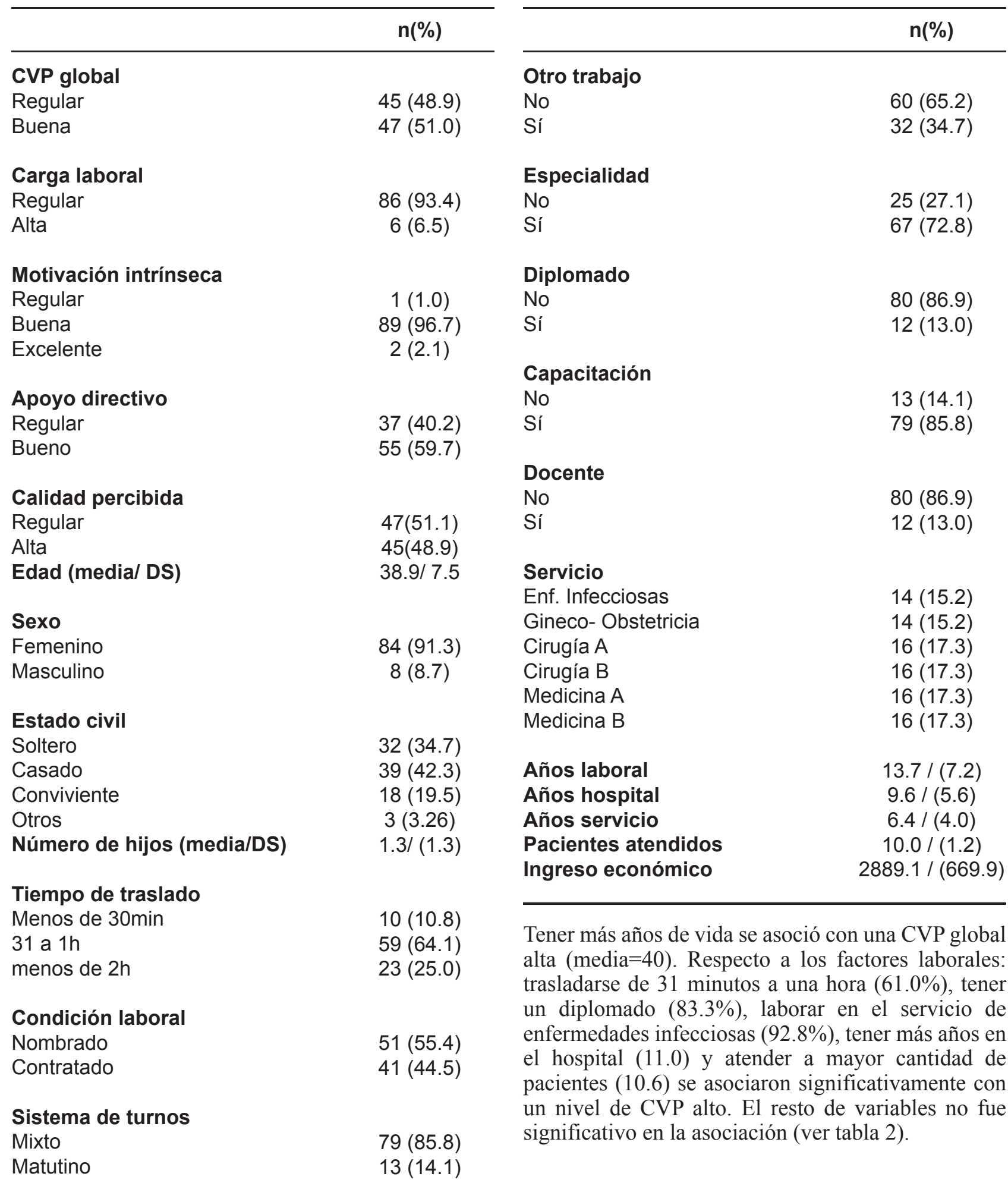

Tabla 1. (continúa) 
Tabla 2. Asociación descriptiva entre los factores y la CVP global

\begin{tabular}{ccc}
\hline & \multicolumn{3}{c}{ CVP global } \\
\cline { 2 - 3 } & \multicolumn{3}{c}{ regular buena } & $\mathrm{p}$ \\
$\%$ & $\%$ \\
\hline
\end{tabular}

Factores sociodemográficos

Edad (media)

Sexo

Femenino

Masculino

Estado civil

Soltero

Casado

Conviviente

Otros

Número de hijos (media)

Factores laborales

Tiempo de traslado

menor igual que $30 \mathrm{~min}$

de 31 a $1 \mathrm{~h}$

menos de $2 \mathrm{~h}$

\section{Condición laboral \\ Nombrado \\ Contratado}

$45.1 \quad 54.9$

$53.6 \quad 46.3$

\section{Sistema de turnos}

Mixto

Matutino

$45.5 \quad 54.4$

$69.2 \quad 30.7$

\section{Otro trabajo}

No

Sí

\author{
Especialidad \\ No \\ Sí
}

Diplomado
No

Sí

\section{Capacitación}

No

Sí

Docencia

No

Sí $\begin{array}{ll}53.7 & 46.2\end{array}$

$16.6 \quad 83.3$

$53.3 \quad 46.6$

$40.6 \quad 59.3$

$\begin{array}{ll}64.0 & 36.0\end{array}$

$43.2 \quad 56.7$

$53.8 \quad 46.1$

$48.1 \quad 51.9$

$51.2 \quad 48.7$

$33.3 \quad 66.6$
Tabla 2. (continúa)

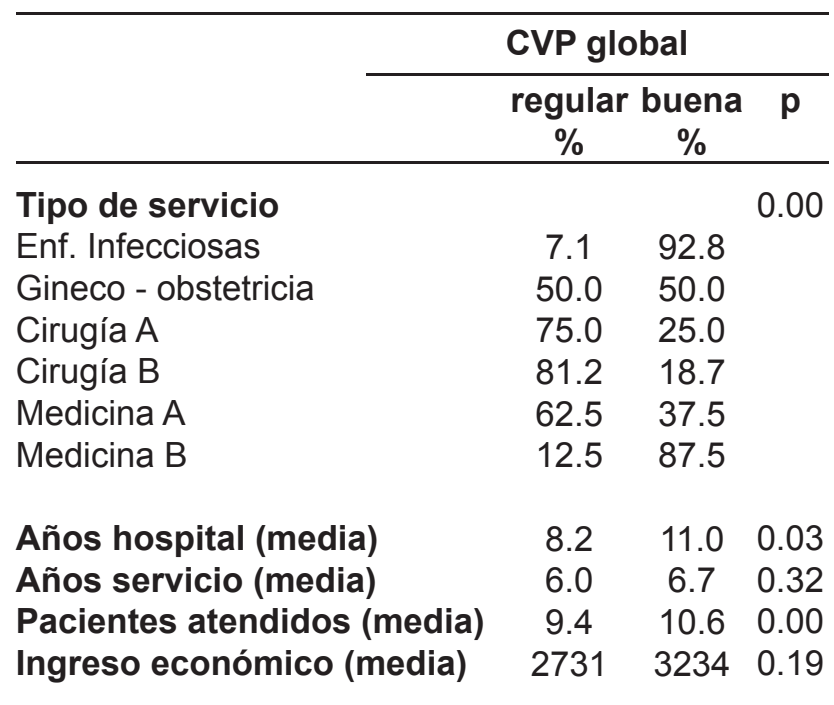

0.02 Respecto a las dimensiones de CVP global: tener menos años de vida, ser soltero, no tener hijos, ser contratado, trabajar en turno matutino, no tener especialidad, trabajar en los servicios de Gineco-obstetricia y Cirugía A, tener menos años

0.41 laborando tanto en el hospital como en el servicio y menos ingresos económicos se encuentran asociados a la carga laboral alta. Asimismo, estar casado y tener un sistema de turnos mixto se encuentran asociados a una motivación intrínseca excelente. Por otro lado, tener más años de vida, tener más hijos, demorar un tiempo de 31 minutos a una hora de traslado de su casa al hospital, tener nombramiento, laborar en un 0.24 sistema de turno mixto, tener especialidad, haberse capacitado en el último año, ser docente, tener más años de experiencia tanto en el hospital como en el servicio, atender a mayor cantidad de pacientes y tener una buena paga se asociaron significativamente con un apoyo directivo alto. Por último, tener más años de vida, demorar un tiempo de 31 minutos a una hora de traslado de su casa al hospital, contar con un sistema de turnos mixtos, tener una especialidad, haber realizado una capacitación en el último año, laborar en el servicio de enfermedades infecciosas, tener más años laborando en el hospital y atender más pacientes por turno se asociaron significativamente a un nivel de calidad percibida alta. Las restantes variables no fueron significativas en la asociación (ver tabla 3). 


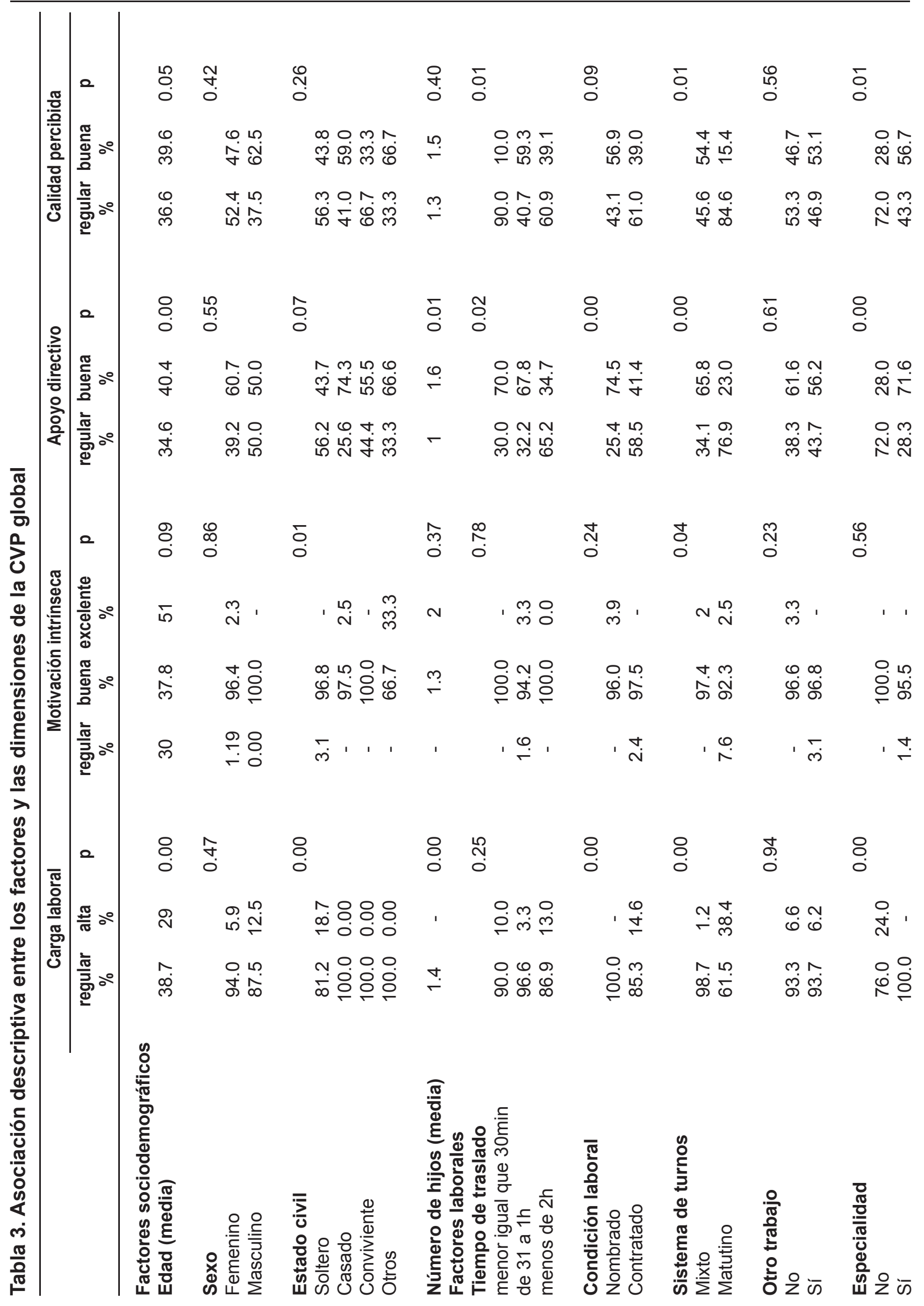




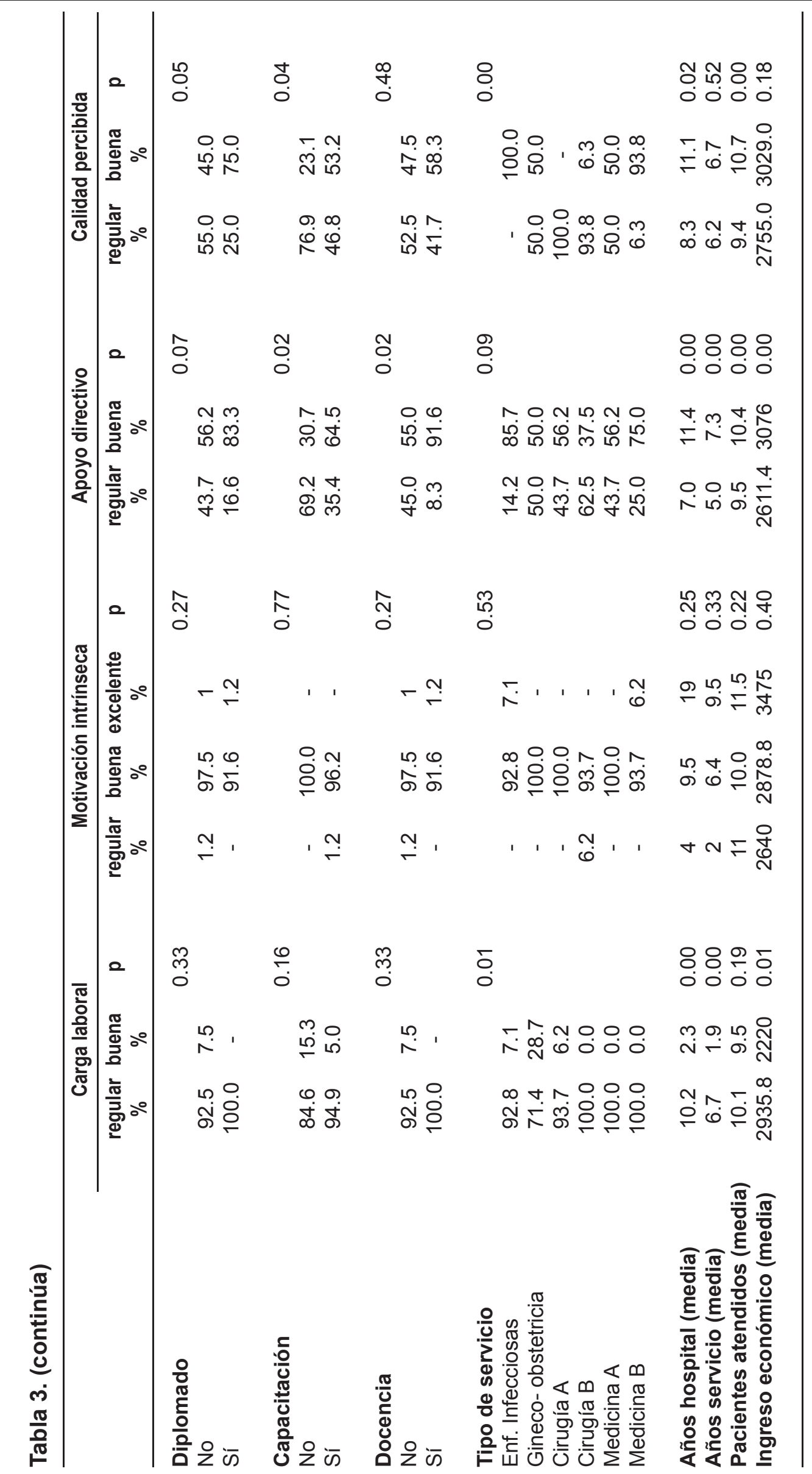


En el análisis de fuerza de asociación los enfermeros que laboran en el servicio Cirugía A tienen menos posibilidades de tener CVP alta en comparación con el servicio de Enfermedades infecciosas $(\mathrm{PR}=0.26)$. Atender a más pacientes se asoció significativamente con CVP global alta. El resto de variables no resultó significativo en la asociación (ver tabla 4).

Tabla 4. Fuerza de asociación entre los factores y la CVP global

\begin{tabular}{lcc}
\hline & RP & $\mathbf{p}$ \\
\hline $\begin{array}{l}\text { Factores asociados } \\
\text { Edad }\end{array}$ & 1.03 & 0.08 \\
Tiempo de traslado & & \\
menor igual que 30min & 1.00 & - \\
de 31 a 1h & 3.05 & 0.12 \\
menos de 2h & 1.95 & 0.39 \\
& & \\
Diplomado & 1.00 & - \\
No & 1.3 & - \\
Sí & 1.00 & 0.10 \\
& & \\
Años hospital & & \\
Tipo de servicio & 1.00 & - \\
Enf. Infecciosas & 0.53 & 0.19 \\
Gineco- obstetricia & 0.26 & 0.02 \\
Cirugía A & 0.20 & 0.01 \\
Cirugía B & 0.40 & 0.07 \\
Medicina A & 0.94 & 0.88 \\
Medicina B & & \\
Pacientes atendidos & 1.39 & 0.00 \\
\hline
\end{tabular}

Respecto a la dimensiones solo hubo asociación significativa para la carga laboral y apoyo directivo. Los enfermeros con más años de vida tienen menos posibilidades de tener una carga laboral alta $(\mathrm{PR}=0.55)$. Los que laboran en un turno matutino tienen 29.38 veces más posibilidades de tener una carga laboral alta en comparación con los que laboran en turno mixto ( $\mathrm{PR}=30.38)$. Laborar más años tanto en el hospital $(\mathrm{PR}=0.43)$ como en el servicio $(\mathrm{PR}=0.45)$ tiene menos posibilidades de tener una carga laboral alta (ver tabla 5). Referente a la dimensión apoyo directivo bueno, tener más años de edad, contar con especialidad y tener mayor antigüedad laboral; e inversamente con ser un profesional contratado se mostraron asociados con esta dimensión (ver tabla 5).
Tabla 5. Fuerza de asociación entre los factores y las dimensiones de la CVP global

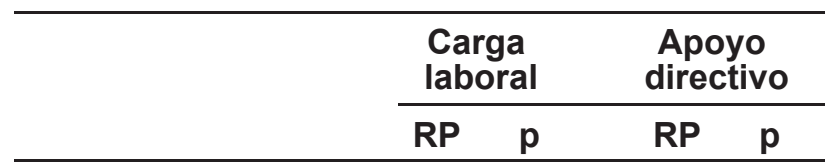

\section{Factores \\ Sociodemográficos \\ Edad}

$\begin{array}{llll}0.55 & 0.01 & 01.03 & 0.02\end{array}$

\section{Factores laborales}

Condición laboral

Nombrado

Contratado

$\begin{array}{llll}1.00 & - & 1.00 & - \\ 2.40 & 0.99 & 0.55 & 0.04\end{array}$

\section{Especialidad}

No

Sí

$\begin{array}{llll}1.00 & - & 1.00 & - \\ 8.19 & 0.99 & 2.55 & 0.02\end{array}$

Sistema de turnos

Mixto

Matutino

$\begin{array}{cccc}1.00 & - & 1.00 & - \\ 30.38 & 0.00 & 0.35 & 0.08\end{array}$

$\begin{array}{lllll}\text { Años en el hospital } & 0.43 & 0.01 & 1.05 & 0.02 \\ \text { Años en el servicio } & 0.45 & 0.04 & 1.05 & 0.08 \\ \text { Ingreso económico } & 0.99 & 0.02 & 1.00 & 0.04\end{array}$

\section{DISCUSIÓN}

En el estudio se encontró que del total de los participantes el $93.4 \%$ presentó una carga laboral regular, el $96.7 \%$ tuvo una motivación intrínseca buena, un $59.7 \%$ tuvo un apoyo directivo bueno y un $51.1 \%$ tuvo una regular calidad de vida profesional percibida. Los enfermeros que laboran en el servicio Cirugía A tienen menos posibilidad de tener CVP global alta. Respecto a la dimensiones, los enfermeros con más años de vida, con más años tanto en el hospital como en el servicio tienen menos posibilidades de tener una carga laboral alta. Los que trabajan en un turno matutino tienen más posibilidades de tener una carga laboral alta. Por otro lado tener más años de edad, contar con especialidad y tener mayor antigüedad laboral; e inversamente con ser un profesional contratado fueron asociados con un apoyo directivo bueno. 
Laborar en el servicio Cirugía A se relaciona con menos posibilidades de tener una CVP global alta. Cabe resaltar que en este servicio la mayoría de los encuestados refirió tener conflictos con otras personas del mismo servicio. Asimismo, en este servicio se atienden a pacientes con cuidados más complejos. Para que un enfermero tenga una CVP alta es necesario tener un buen clima organizacional, incluyendo apoyo por parte de su equipo de trabajo o de sus superiores y buenas relaciones con sus compañeros (12). Situaciones contrarias conllevarían a consecuencias negativas. Por otro lado, es necesaria una buena dotación de enfermeros cuando se trata de cuidar a pacientes con cuidados más complejos (17). Un aumento de la edad fue un factor protector de la carga laboral alta. Un estudio reporta un resultado similar (13). Esto podría explicarse porque los enfermeros con más años de vida tienen menos quejas respecto a sus condiciones laborales, ya que la mayoría de ellos tienen un contrato seguro e incluso se sienten más motivados y autónomos por la experiencia laboral que han adquirido durante los años (13). Otro hallazgo del estudio muestra que tener más antigüedad tanto en el hospital como en el servicio resultan ser factores protectores de la carga laboral alta (15). Los enfermeros con más antigüedad laboral en el hospital y servicio perciben menos la carga laboral debido a la experiencia que han adquirido (18).

Contar con un sistema de turnos matutinos influye para tener una carga laboral alta. La turnicidad facilita el estrés debido a una excesiva cantidad de trabajo (19). Los enfermeros que trabajan turnos diferentes mejoran su tolerancia al trabajo debido a la adaptación del sueño (20). Igualmente los turnos matutinos del hospital del enfermero asistencial son más laboriosos por la cantidad de actividades propias de esos horarios (14). En algunos casos esta situación podría llevar a problemas psicológicos como el estrés y ansiedad somática (14).

Respecto a la dimensión apoyo directivo de CVP tener especialidad está asociado con tener un apoyo directivo alto, mientras que ser contratado se asocia con un apoyo directivo bajo. Existen estudios que reportan que los enfermeros especialistas son apreciados por su habilidades y capacidades por parte del equipo interdisciplinario y supervisores de la directiva de la institución que laboran, ya que demuestran una mayor eficacia en los cuidados de los pacientes $(21,22)$. El personal contratado tiene menos probabilidad de recibir un apoyo directivo alto. La literatura menciona que los trabajadores contratados experimentan menor satisfacción laboral que los trabajadores permanentes o nombrados (23). Esto se debe a que los últimos tienen un contrato fijo, un compromiso organizacional sostenido y por ende cuenta con un apoyo directivo (24).

Hay algunas limitaciones del estudio respecto al cuestionario empleado ya que el mismo mide solo percepciones. Sin embargo, es un buen punto de partida para conocer la CVP aunque no mide las expectativas, las condiciones reales de trabajo (ruido, carga física y mental, y autonomía) de los enfermeros. Estos puntos deben ser considerados en futuros estudios.

\section{CONCLUSIONES}

Laborar en el servicio Cirugía A está asociado con la CVP global baja. Asimismo, se encontró asociación significativa entre los factores laborales $y$ las dimensiones de la CVP global. Tener más años de vida, laborar más años tanto en el hospital como en el servicio resultaron protectores para una carga laboral alta. Sin embargo, trabajar en un turno matutino se asoció igualmente con una carga laboral alta. Por otro lado tener más años de edad, contar con especialidad y tener mayor antigüedad laboral fueron asociados con un apoyo directivo bueno.

Estos resultados contribuyen al desarrollo de estrategias por parte de las autoridades de las instituciones y del personal de enfermería mismo para mejorar la calidad de vida profesional. Las estrategias deberían estar encaminadas a la mejora del apoyo directivo tanto a contratados como nombrados, la organización considerando el número de pacientes y la satisfacción laboral en modo tal que repercutan positivamente en la salud de los enfermeros y por ende brinden una atención de excelencia a los pacientes que son el motivo principal del profesional de enfermería. 


\section{REFERENCIAS BIBLIOGRÁFICAS}

1. Fernández A, Clotilde E; Casado del Olmo M. Calidad de vida profesional de los profesionales de enfermería en atención primaria de Soria. Biblioteca Lascasas. 2007;3(1).

2. Hanzeliková A, López G, Pomares M, Pardo M. J, \& Paz M. La calidad de vida profesional de las enfermeras en geriatría. Enfermería Global. 2011;10(24).

3. Organización Mundial de la Salud. Perfil mundial de los trabajadores sanitarios. En: El Informe sobre la salud en el mundo 2006-Colaboremos por la salud. Ginebra: OMS; 2006.

4. Martín J, Cortés J, Morente M, Caboblanco M, Garijo J, \& Rodríguez A. Características métricas del cuestionario de calidad de vida profesional (CVP-35). Gac Sanit. 2004;18(2):129-136.

5. Sosa O, Cheverría S, Rodríguez E. Calidad de vida profesional del personal de enfermería. Rev Enferm Inst Mex Seguro Soc. 2010;18(3):153-158.

6. Cañón S, Galeano G. Factores laborales psicosociales y calidad de vida laboral de los trabajadores de la salud de ASSBASALUD ESE Manizales (Colombia). Archivos de medicina. 2011;11(2):114-126.

7. Moradi T, Maghaminejad F, Azizi-Fini I. Quality of Working Life of Nurses and its Related Factors. Nurs Midwifery Stud. 2014;3(2): e19450.

8. Sancho S, Vidal C, Cañellas R, Caldés M, Corcoll J, Ramos M. Análisis de situación y propuestas de mejora en enfermería de atención primaria de Mallorca: un estudio con grupos focales. Rev. Esp. Salud Publica. 2002;76(5):531-543.
9. Melita A, Cruz M, Merino J. Burnout en profesionales de enfermería que trabajan en centros asistenciales de la octava región, Chile. Cienc enferm. 2008;14(2):75-85.

10. Arroyo J. Los sistemas descentrados de recursos humanos en salud: el caso del Perú, 1990-2005. Ciência \& Saúde Coletiva. 2006;11(4):1063-1072.

11. Maticorena J, Beas R, Anduaga A, Mayta P. Prevalencia del síndrome de burnout en médicos y enfermeras del Perú, ENSUSALUD 2014. Revista Peruana de Medicina Experimental y Salud Pública. 2016;33(2):241-247.

12. Novoa M, Nieto C, Forero C, Caycedo E, Palma M, Montealegre M, et al. Relación entre perfil psicológico, calidad de vida y estrés asistencial en personal de enfermería. Universitas psychologica. 2005;4(1):63-76.

13. Ordaz E, Ronda E. Salud y condiciones de trabajo en trabajadores mayores. Medicina y Seguridad del Trabajo. 2015;61(240):314-324.

14. Gutiérrez E, Loredo M, Manzano R. Calidad de vida profesional del personal de enfermería del hospital del niño y la mujer en San Luis Potosí [tesis]. San Luis Potosí: Universidad Autónoma de San Luis Potosí. Facultad de Enfermería; 2010.

15. Albaladejo R, Villanueva R, Ortega P, Astasio P, Calle E, Domínguez V. Síndrome de Burnout en el personal de enfermería de un hospital de Madrid. Revista española de salud pública. 2004;78(4):505-516.

16. Grimaldo M, Reyes M. Calidad de vida profesional y sueño en profesionales de Lima. Rev Latinoam Psicol. 2015;47(1):50-57.
17. Castrillon M. Pensando en la formación de futuros profesionales de enfermería en América Latina. Investigación y Educación en Enfermería. 2008;26(2):114-121.

18. Risquez R, Hernández P, Fernández G. Burnout y salud percibida en profesionales de enfermería de Cuidados Intensivos. Enfermería intensiva. 2008;19(4):169-178.

19. Martínez C, López G. Características del síndrome de burnout en un grupo de enfermeras mexicanas. Arch Med Fam. 2005;7(1):6-9.

20. Deschamps A, Olivares B, Zabala R, Asunsolo Á. Influencia de los turnos de trabajo y las guardias nocturnas en la aparición del Síndrome de Burnout en médicos y enfermeras. Medicina y Seguridad del Trabajo. 2011;57(224):224-241.

21. Barrett L, Yates P. Oncology/haematology nurses: a study of job satisfaction, burnout, and intention to leave the specialty. Australian Health Review. 2002; 25(3):109-121.

22. Hall D. The relationship between supervisor support and registered nurse outcomes in nursing care units. Nursing Administration Quarterly. 2007;31(1):68-80.

23. Wilkin L. I can't get no job satisfaction: Meta-analysis comparing permanent and contingent workers. Journal of Organizational Behavior. 2013;34(1):47-64.

24. Jalonen P, Virtanen M., Vahtera J, Elovainio M, Kivimaki M. Predictors of sustained organizational commitment among nurses with temporary job contracts. Journal of Nursing Administration. 2006;36(5):268-276. 


\section{Factors associated with professional quality of life in nurses at a public hospital}

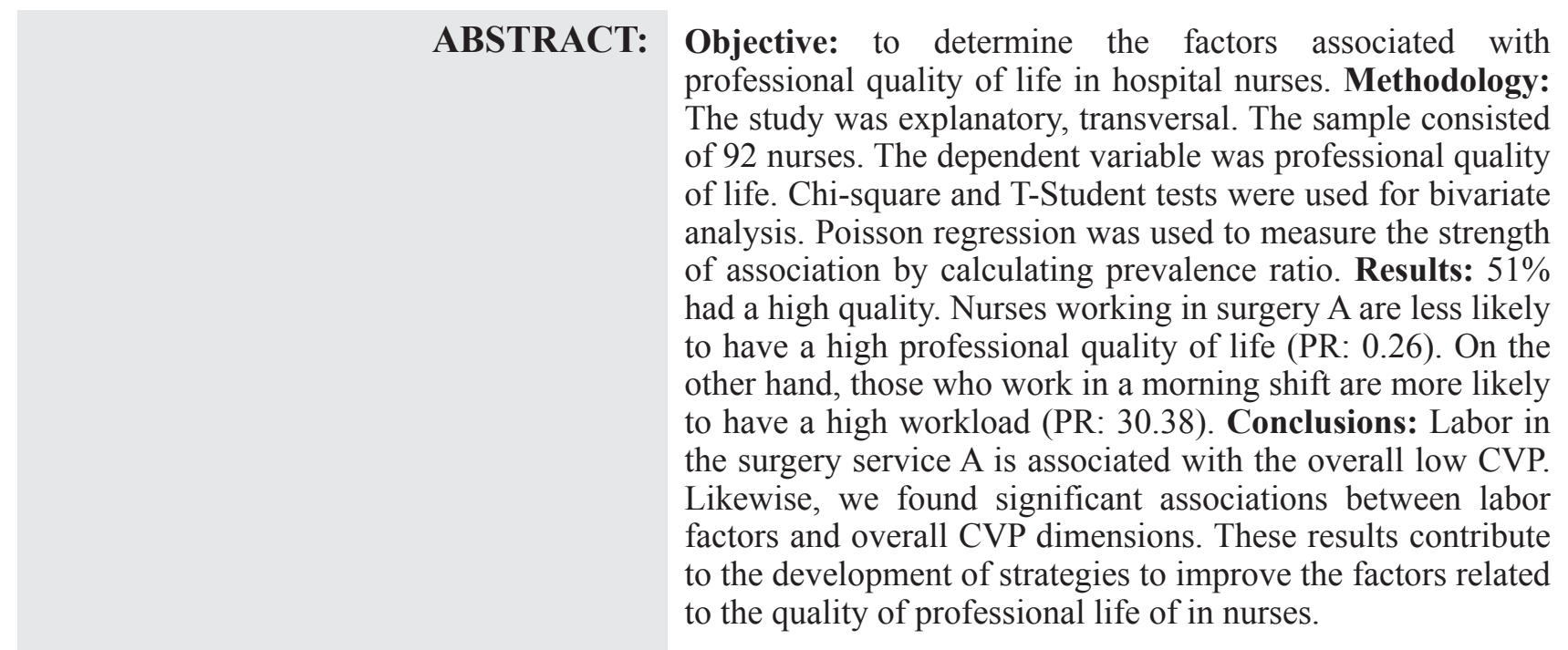

KEY WORDS: Quality of Life; Nurses; National Strategies. 